\title{
A Method for Integrating Process Description and User Interface Use during Design of RIA Applications
}

\author{
Hernán Casalánguida and Juan Eduardo Durán \\ Facultad de Matemática, Astronomía y Física, Universidad Nacional de Córdoba, Medina \\ Allende s/n, Córdoba, Argentina \\ hcasalan@hal.famaf.unc.edu.ar, duran@mate.uncor.edu
}

\begin{abstract}
During design of rich internet applications (RIA) it is important to integrate in one model the description of both the interactions of the user with the system, and the processes (considering data flow, control flow, and possibly, detailed autonomous task descriptions), for having a very rich set of relations between modeling elements that can be inspected. Such integrated models consider both UI aspects, and user interaction aspects; therefore, their construction needs the participation of both graphic designers and analysts, leading possibly to communication problems, less productivity, and perhaps to introduce errors. To treat these problems, we propose a method consisting of: process requirements description, traces definition from requirement elements into either UI elements (UIE)/events on UIEs (provided by UI designers), or to autonomous actions design (provided by analysts); in addition, we present a transformation from the deliverables of this process onto an integrated model.
\end{abstract}

\section{Introduction}

There exist in the literature some RIA methods considering notations that treat in the same diagram both aspects of the UI, and aspects related with the interaction: WebML [1,2], notations based on state machine concepts (see OOH4RIA [3], ADVs [4]), and notations considering event condition action (ECA) rules (see RUX [5], OOWS 2.0 [6], MARIA [7]). In [8] it is said that "A general limit of ECA rules is that they do not always reflect the procedural, imperative way of thinking familiar to many people from imperative or object-oriented programming". State Machines are much better to understand control flow, but they are not much adequate for describing ECA rules (see [9], page 16, for an explanation). Therefore, a modeling notation allowing to express data and control flow, ECA rules, and possibly to describe UI elements will be useful for expressiveness and understandability.

In [6] (page 78) it is stated that: "to treat in the same model together visual aspects of the UI (e.g. its organization in terms of UIEs) with aspects related with the interaction (e.g. the events that initiate the communication with the system, or the information to introduce) has as a direct consequence more complex models that need to be defined by both analysts and graphic designers". To build such kind of models without considering an appropriate development process may lead to the following 
problems: inconsistency introduction and/or the construction of such models by analysts and UI designers working at the same time. These problems are important, because complex models tend to be more difficult to understand, and problems of the interaction between analysts and UI designers may impact productivity and the quality of the product.

Integrated modeling notations contemplating both user interaction in the UI and process description also consider both UI aspects and user interaction aspects, with the addition of process aspects (i.e. data flow, control flow, and, possibly, detailed autonomous action description - i.e. tasks executed by the system without user participation); therefore, their construction has the same kind of problems. Hence, it is necessary to simplify in some ways such kind of modeling notations, and to have an appropriate development process to construct models respecting them to avoid problems concerning the interaction of both analysts and UI designers.

To construct models comprising user interaction in the UI and process description, we propose the following development process to treat the described problems: First, to use a requirements model for describing the process that must be respected by both graphic designers and analysts. Next, the data mentioned in the requirements model is designed in detail and must be known by graphic designers and analysts, because such data impact both the UI and autonomous actions considered by analysts. Following, a design notation is used by graphic designers to describe UIEs and event patterns on them, and simultaneously analysts use a design notation for the definition of autonomous actions. Finally, from these three notations a model integrating the processing of events over the UI with processes is automatically generated.

Our proposal is possible if we start from our UML activity diagram (AD) requirements notation for RIAs in [10]: such ADs have modeling elements that need to be described in design by UI designers (concerning output, input request, and input); there are also other modeling elements in them that need to be described in design by analysts (i.e. autonomous actions); and there are also modeling elements that refer to the data managed by the application (i.e. pins and object nodes) that need to be described in detail in design.

The minimum information necessary to generate a model integrating UI use and process description is: an $\mathrm{AD}$ using our notation in [10] for describing process requirements, and trace relationships of two kinds: a) traces between these AD's actions and design elements that can be either UIEs, or event patterns, or activities for autonomous actions; b) traces between AD's object nodes and design elements for representing data in detail. In this paper we assume that we have available this information, and we are not worried about how to obtain these trace relationships.

In this work, we present a development process that starts with RIA requirements modeling using the UML profile in [10] (see Sec.2). Next, actions related to input and output in such ADs are associated with either UIEs or events (to provide more details about them). UIEs respect a UML profile called RIAAD presented in Sec. 3; RIAAD consists of two notations: one extending class diagrams to represent UIE, and another extending UML ADs for describing autonomous actions. Finally, these ADs and their trace relationships are mapped onto user interface use and process description models (UIUPDM); these transformations are implemented in ATL, and presented in Sec. 5. 


\section{Activity Diagrams for Rich Internet Application Requirements}

In [10] an action inside an $\mathrm{AD}$ for a use case (UC) can be of one the following stereotypes: «search» (it represents database queries), «job» (it represents a call behavior action, whose activity performs an autonomous action), «input» (it represents the provision of an input by an human actor), «output request» (it represents the request by the system for the provision by a human actor of some inputs), «output content» (it represent the system displaying content), «input and suggest» (it represents the provision of an input by a user, and the use of automatic suggestions for input that are selectable), and «output message» (it represents the system showing a message to the user - e.g. a message of error, success, status, a help, or warning). In addition, we consider the stereotype: «output media» (the system shows content by playing a media file - e.g., image, video, audio, animation, and presentation - and also shows the controls of the player for user interaction). Icons: ○ («search»), («job»), Ð («input»), ? («output request»), and («output message»).

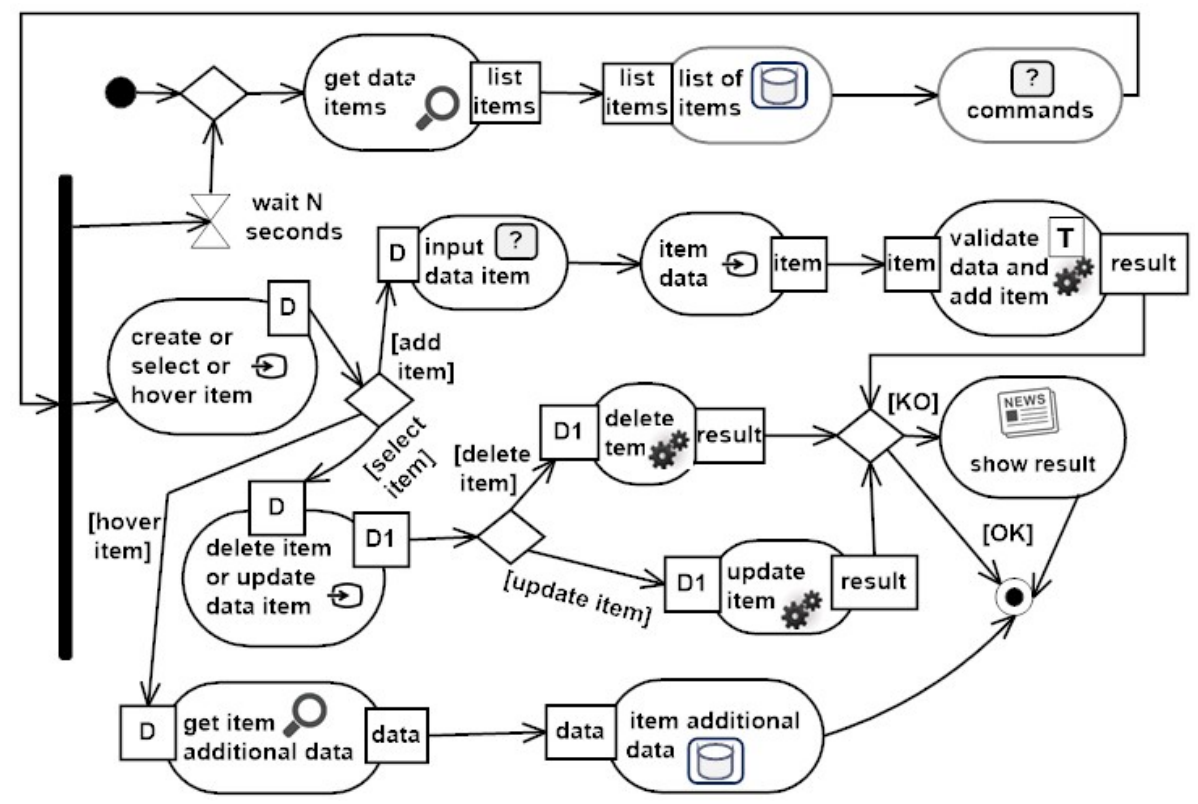

Fig. 1. UC Enriched CRUD of items of a list

Fig. 1 shows an AD for an enriched CRUD of items of a list UC http://rirand.com/blog/jqgrid/jqgrid.html is an example of such a kind of application. Some of the features of this UC are: it permits collaborative edition of the list, because the list is redisplayed every $N$ seconds $(\mathrm{N} \leq 5)$ showing the changes in the list 
made by other people; item registers can be updated by editing their fields; by hovering an item of the list you can read additional data about it.

\section{RIA Application Abstract Design (RIAAD)}

In this section we present the RIAAD notation, consisting of design elements that will be used when defining trace relationships from requirement's actions. Our desire is that RIAAD for UIE description have a rich set of UIEs to allow design decision taking; this part of RIAAD, to our knowledge, fills some gaps we have found in existing abstract design notations for RIA: the representation of editable UIEs, being them either elementary or content structures; the abstraction of special UI patterns for navigation in RIA like breadcrumb, alphanumeric filter links, and navigation bar, and the appropriate representation of suggest field; the representation of UIEs for the edition of multimedia objects (audio, video, etc.), and of documents -i.e. presentations, spreadsheets - (e.g. youTube allows editing videos https://www.youtube.com/editor, and Google Drive allows editing documents). RIAAD part for UIE modeling is based on UWE [11], UWE-R [12], and MARIA [7] metamodels, with some additions necessary to fill the mentioned holes.

The UIEs of RIAAD are presented in Figs. 2 and 3. A UiStructure respects some structuring rule, and are classified into: ContentStructure, AccessStrucure, UiInputStructure, and UiOutputStructure. ContentStructure (CS) represents an UIE with a structure used for content presentation. A CS can be editable (i.e. allowing the edition of some of its contents) or not (it is only used to present content to the user). CSs are classified into: List, Table, Tree, and Record.

AccessStructure represents an UIE used for accessing other UIEs (i.e. navigation) or performing an action. LinksBased represents a link grouping to access either other UIEs or performing an action; it is classified into: Menu, Breadcumb, and ANFilterLinks. Breadcumb represents a UIE containing a list of steps; each step has a link, a name, and a level number. Breadcumbs are used to represent navigation paths, whose nodes can be visited by selecting steps. ANFilterLinks: (alphanumeric filter links) represents a list of items ordered alphanumerically; each item has a link, and an alphanumeric character as name. ANFilterLinks are used to organize a set of named objects $S$ in the following way: by selecting an item $i$, we access to the objects whose identifier starts with the name of $i$. NotLinksBased represents access to UiStructures and/or functionality not based exclusively on links; for some NotLinksBased structures the user may fill input structures - e.g. forms - for gathering of parameters for UiStructure generation or functionality execution. We considered three kinds of NotLinksBased elements: NavigationBar, NavList and NavAltBlocks.

NavigationBar represents a set of Anchors and Forms (at least one form must be present). Usually a navigationBar behaves in the following way: after a Press event (i.e. anchor selection or form submission) a UiStructure is shown, or a command is executed. For instance, see the last line of site http://www.vodahost.com/partner/.

NavList (navigation list) represents an UIE containing a set of items; each item contains: optionally an anchor corresponding with content displayed for this item, optionally a navigationBar for parameters providing and/or functionality access, and one or more BasicUIElements for describing an item. Typical examples of NavList 
are indexes (only the navigationBar is excluded), inbox in Gmail (NavigationBar containing an UiInputStructure with only checkboxes, and anchors to mails), Koha library management system (http://manual.koha-community.org/3.6/en/opac.html) bibliographic search the catalog functionality (a NavigationBar consisting of a check box, and of forms and anchors for accessing commands; and an anchor to a bibliographic record). NavAltBlocks represents an object containing a collection of Blocks in which only one block at a time is visible; each block contains one or more UiStructures, has a name, and an order.

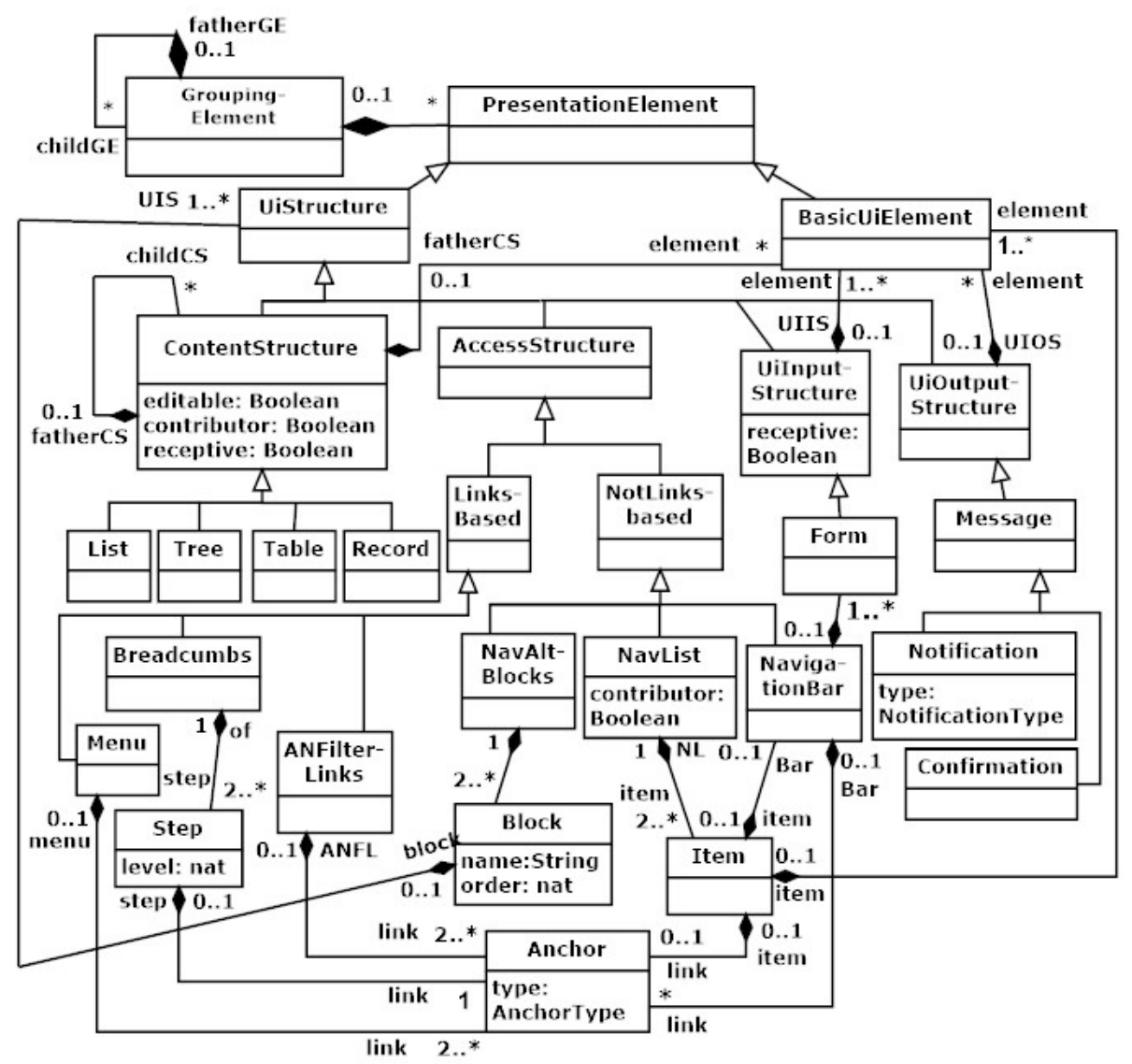

Fig. 2. UI element classification

UIInputStructure represents a UIE used for user input; a special kind of UiInputStructure is a form; a UiInputStructure contains BasicUiElement instances with typeOfEdition="input". UIOutputStructure represents a UIE used for presenting information to the user, and contains BasicUiElements having typeOfEdition="noeditable". A kind of UiOutputStructure element is Message, and is classified into: Notification (e.g. of some event) and Confirmation (i.e. a confirmation request). 
A CS/NavList can be a contributor (it can pvovide elements to a CS/UiInputStructure). A CS/UiInputStructure can be receptive (it can receive elements from other CS/NavList).

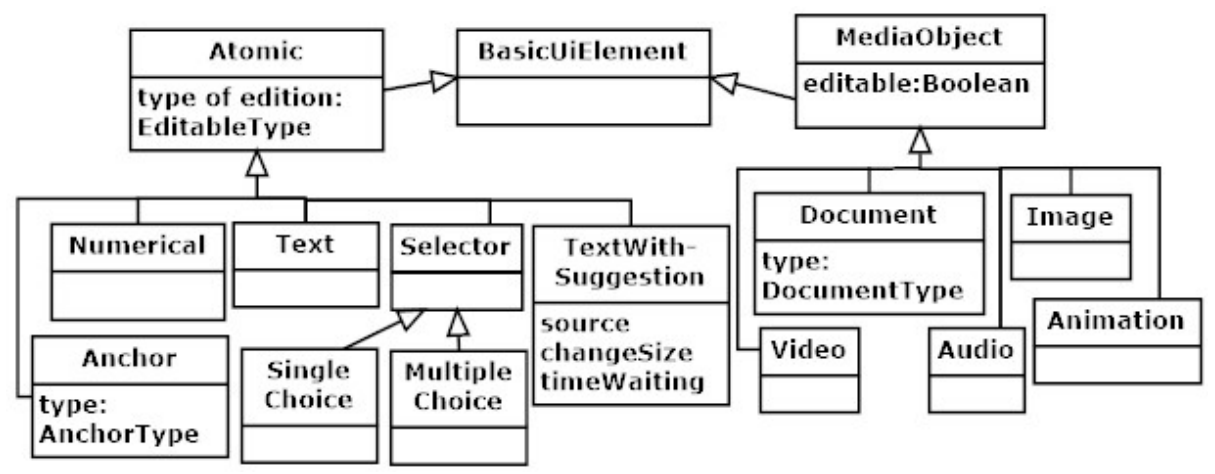

Fig. 3. Classification of BasicUiElement

Atomic elements can be used for different purposes according to their type of edition: input (for information input), editable (for information editing), and no_editable (for the presentation of information in a specific modality). textWithSuggestion represents editing text with suggestion (its type of edition can be editable or input) and has as tagged values: search (the value is the name of a search action), change size (values can be "char"। "word"। "sentence"), time waiting - i.e. before suggesting - (the value is in Nat; 0 means not waiting). AnchorType can have as values: classicalLink (link to another web page/document), bookmarkLink (a link to a position in the same web page), and commandLink (its selection initiates an action or task). MediaObject represents a media file; if a mediaObject is editable then it also includes the edition widget according to the specific media; else it only represents the playing of a media file. We considered mediaObjects of kinds: document, image, video, audio and animation. DocumentType can have as values: PlainText, RichText (an enriched text document), Presentation (a document consisting of slides), SpreadSheet.

All the above UIEs with the exception of UiComponent, PresentationElement, UiStructure, and BasicUiElement are stereotyped, extend Class, and their stereotype names are: “《”++ element name ++ “»".

An atomic event consists of its name, its source (an UIE instance), and its data. We assume that in any given time of a web application execution, there exists a stream of the atomic events that happened; in addition, for each atomic event in the stream there is a time stamp of its occurrence. Table 1 shows the atomic event classification considered in this paper. For each event type we list the event contained in it and the UIEs that act as sources of such events. An event pattern (EP) represents a set of events respecting the pattern and can be used for querying the stream of atomic events (i.e. a list of events that belong to the event stream can respect or not an EP). Now we provide a BNF grammar for event patterns. An atomic EP consists of: a name of event, the source, and optionally event data. A composite EP is described by providing all composition operations (given by non-terminals $<\mathrm{OP}>$ and $<\mathrm{REP}>$ ) and atomic events participating. The composition operators were taken from $[13,14]$. 


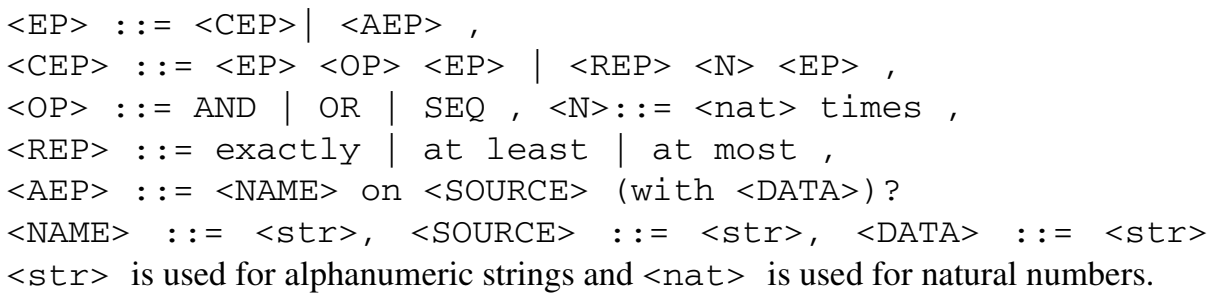

Table 1. Atomic Event Classification

\begin{tabular}{|l|l|l|}
\hline \multicolumn{1}{|c|}{ Event Type } & \multicolumn{1}{|c|}{ Event name } & \multicolumn{1}{c|}{ Source of the event } \\
\hline \multirow{4}{*}{$\begin{array}{l}\text { User interface } \\
\text { events }\end{array}$} & Press & Anchor, Form (submission) \\
\cline { 2 - 3 } & Over, moveOut & BasicUiElement, elements of CS. \\
\cline { 2 - 3 } & enter, edited & CS (element edition), BasicUiElement \\
\cline { 2 - 3 } & select & $\begin{array}{l}\text { LinksBased, Selector, Selector in Form. } \\
\text { Block in NavAltBlocks. }\end{array}$ \\
\cline { 2 - 3 } & remove, add, move & CS (modification) \\
\cline { 2 - 3 } & select to share & contributor CS/NavList \\
\cline { 2 - 3 } & Put & receptive CS/UiInputStructure \\
\hline Data events & update, delete, insert, & relations, XML document. \\
\hline Transaction & commit, abort, request & Transaction \\
\hline Timer events & start, timeout & Timer \\
\hline Service events & start, finish & web service \\
\hline $\begin{array}{l}\text { Developer } \\
\text { defined }\end{array}$ & Given by the developer & $\begin{array}{l}\text { The actual web application or an external } \\
\text { application. }\end{array}$ \\
\hline
\end{tabular}

«event»- 4- extends Action and represents an event list occurrence in the event stream respecting an EP. An «event» action has a tagged value called expr, whose value is an EP expression or an identifier of an EP expression; in addition, an «event» action has an output pin whose tokens can represent an event list matching the EP or data of the event list.

Now, we describe the part of RIAAD consisting of elements to be used for describing «job» Actions. To our knowledge, our classification of actions for modeling autonomous actions not only serves for changing UIE content values (like OOWS 2.0 [6], and RUX [5]), but also adds facilities for automatically change the structure of UiInputStructures and add/delete elements in CS, and we introduced «data process» actions. To describe «job» Actions with an activities, we consider the

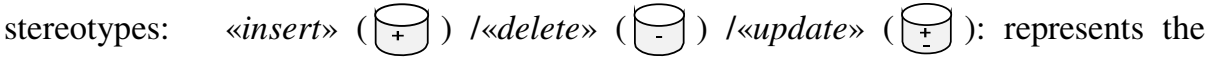
insertion/ deletion/update of either data elements, or relations between data elements, or a view of a database (e.g., a relational database or XML document). «insertUI» $(+)$ / $($ deleteUI» $(\square)$ : represents the insertion/deletion of either an element inside a UI CS. «updateUI» ( \pm ): represents the automatic update by the application of information of either a CS, or a UIBasicElement, or a UIBasicElement in a 
UiInputStructure. For changing the structure of a UiInputStructure (i.e. adding and removing some UiBasicElements and maintaining the rest with the same values) we use actions with setereotype «change UiIS». «dataRetrieve» (䖵): represents the search of information from a given source that can be a text file, a relational database, an XML document, a view of a database. «getUIContent» $(\square)$ : represents the collection of content from one or several UI elements. «UIPropertyChange» $(\square:=)$ represents an action that alters a UIE properties (e.g., accessible - i.e. if the UIE is visible, audible, etc. depending on the modality -, enabled - i.e. is true if and only if events on the UIE are enabled by the system-, and focus - UIE on focus -; it has a tagged-value property, whose value is the property name, and a value tagged value, whose value is the property's value. «validation» $(\sqrt{ } \mid \times)$ : represents the validation of information or business rules (in the client side - e.g. UIE content validation - or in the server side). «dataProcess»: it represents information processing (e.g. data coming from a database, content coming from an UIE). «generateEvent»: it represents the generation of an atomic event over a UIE; it has a tagged value expr, with an atomic EP expression as value. «external job»: represents the invocation of either an external task (e.g. the invocation of a web service or the treatment of an application generated event by another application), or the collection of information not belonging to the application (e.g. mashups). «external notification»: represents a notification to the application coming from an external job (e.g., a web service notification message, or the notification of an event from an external application to be processed).

\section{Relating Actions in an AD UC with Modeling Elements}

Actions whose stereotypes start with the word "output" are associated with UIEs (e.g. from RIAAD metamodel). An «input» action is associated usually with an «event» action representing the occurrence of an EP. A «job» action is associated with an AD describing the activity performed by the job and using the stereotypes explained at the end of Sec. 3. A «search» action is associated with a «data retrieve» action.

Fig. 4 shows the associations to some actions of the AD in Fig. 1 with UI elements and with «event» actions: «output request» commands is associated with an «UiInputStructure» commands containing three «anchor» elements of type commandLink; action «output content» list of items is associated with «List» items being editable; «input» create or select or hover item is associated with «event»create or select or hover, where $E$ is the identifier of the expression: Press on create item OR enter on items OR Over on items; «input» delete item or update item data action is associated with «event» delete or update item, where $\mathrm{E}_{1}$ is the identifier of the expression: Press on delete item OR (change on item SEQ Press on update item); and action «output request» input item data is associated with «Form» new item popup.

«job» validate data and add item is associated with the activity described in Fig. 5: first, the information about the item is validated; next, a checking for business rules violation is performed; following, item is inserted into the database, and finally, the presentation of the items list is updated to reflect the item insertion. 


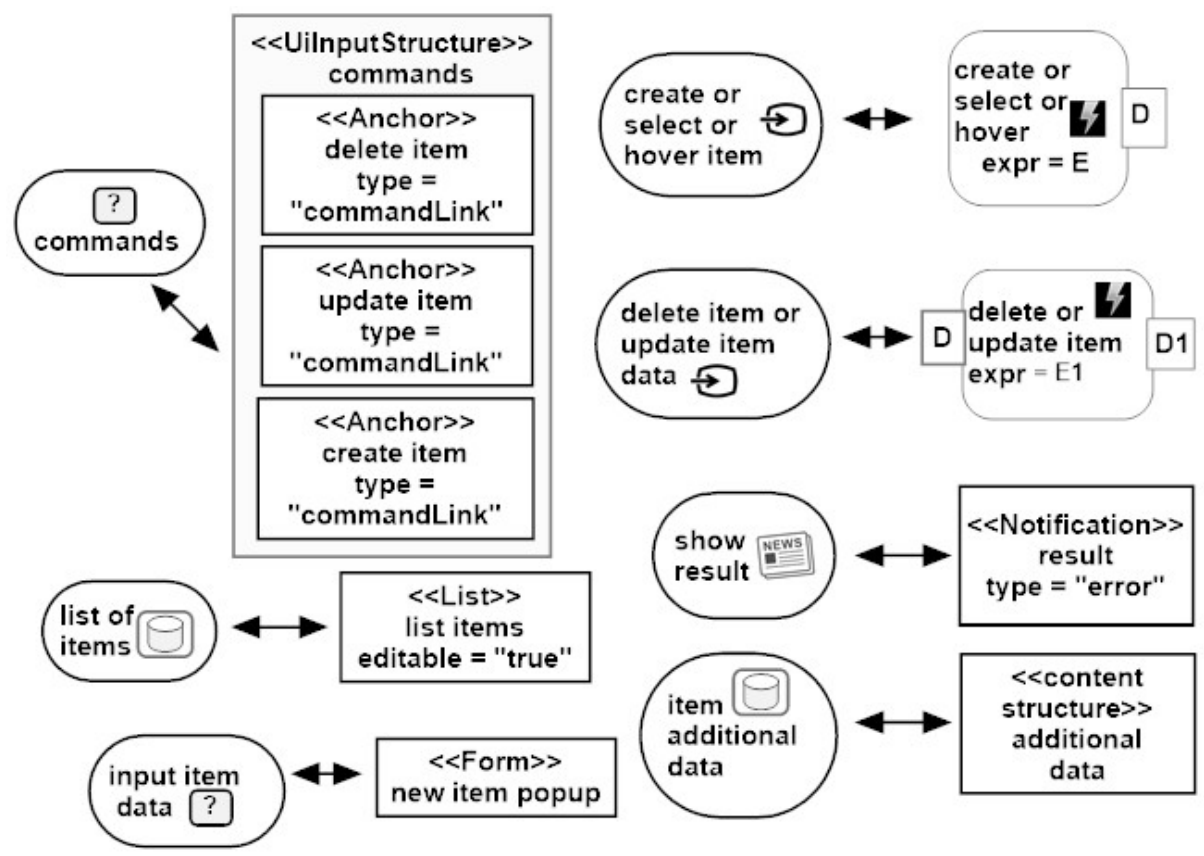

Fig. 4. Association of design elements to actions for CRUD operations on lists

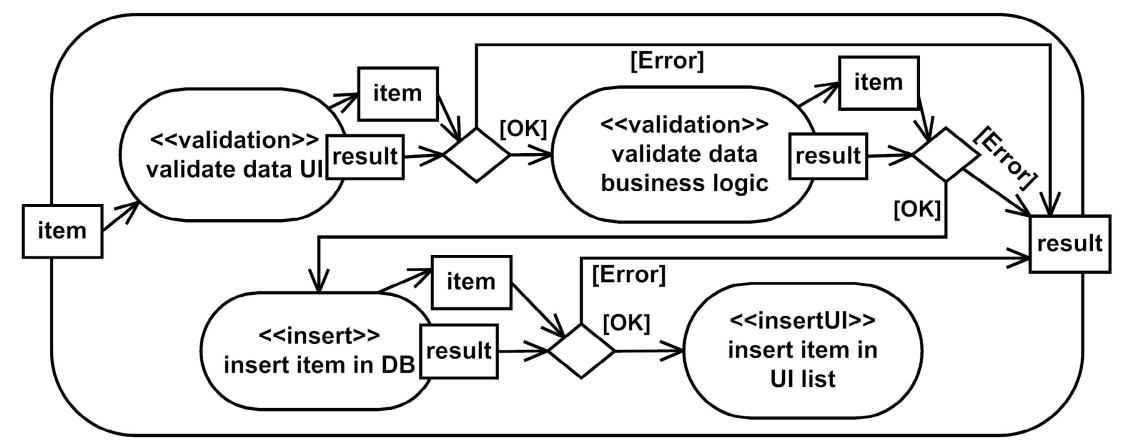

Fig. 5. Refinement of validate data and add ítem job

\section{User Interface Use and Process Description Models (UIUPDM) and Their Automatic Generation}

At least an UIUPDM should describe which UI components are presented and when, and have «event» actions with meaningful names; to have less than that is too few, because we need to talk about UI use. For this purpose, we consider the following stereotypes: «synch» $(\rightarrow \square)$ extends Action, and represents the synchronization of some data with an existing presentation element - to present this data-; for the 
representation of the data we use inputPins; the name of a «synch» action has the form "to" $++P E$, where $P E$ is a UIE name; in addition, if $P E$ is not yet visible then it is shown. "present» (口) extends Action, and represents the presentation of UIEs that do not exhibit content coming from object nodes.

For the definition of UIUPDMs we decided not to show EP expressions inside the diagrams to make them less overcharged. We suggest instead writing EPs associated to identifiers in event actions of a UIUPDM model outside the diagram. In addition, we decided not to show in a UIUPDM the description of objects/data structures associated with tokens for object nodes. We consider three kinds of UIUPDMs: a) to show job descriptions, and avoid showing UIE descriptions; (this is useful when there are actions inside job descriptions that access/modify the UI); b) To show UIE descriptions, and avoid showing job descriptions (it is easy to inspect descriptions about user interaction in the UI, and UIEs presentations); c) to show details only of UIEs and job descriptions of small size. We propose to be able to generate automatically the three kinds of UIUPDM models; the user needs only to decide, which possibilities are appropriate for the situation at hand. In this section we will illustrate in detail the automatic generation of UIUPDM case a), and for the other two cases we only explain how to define the transformation.

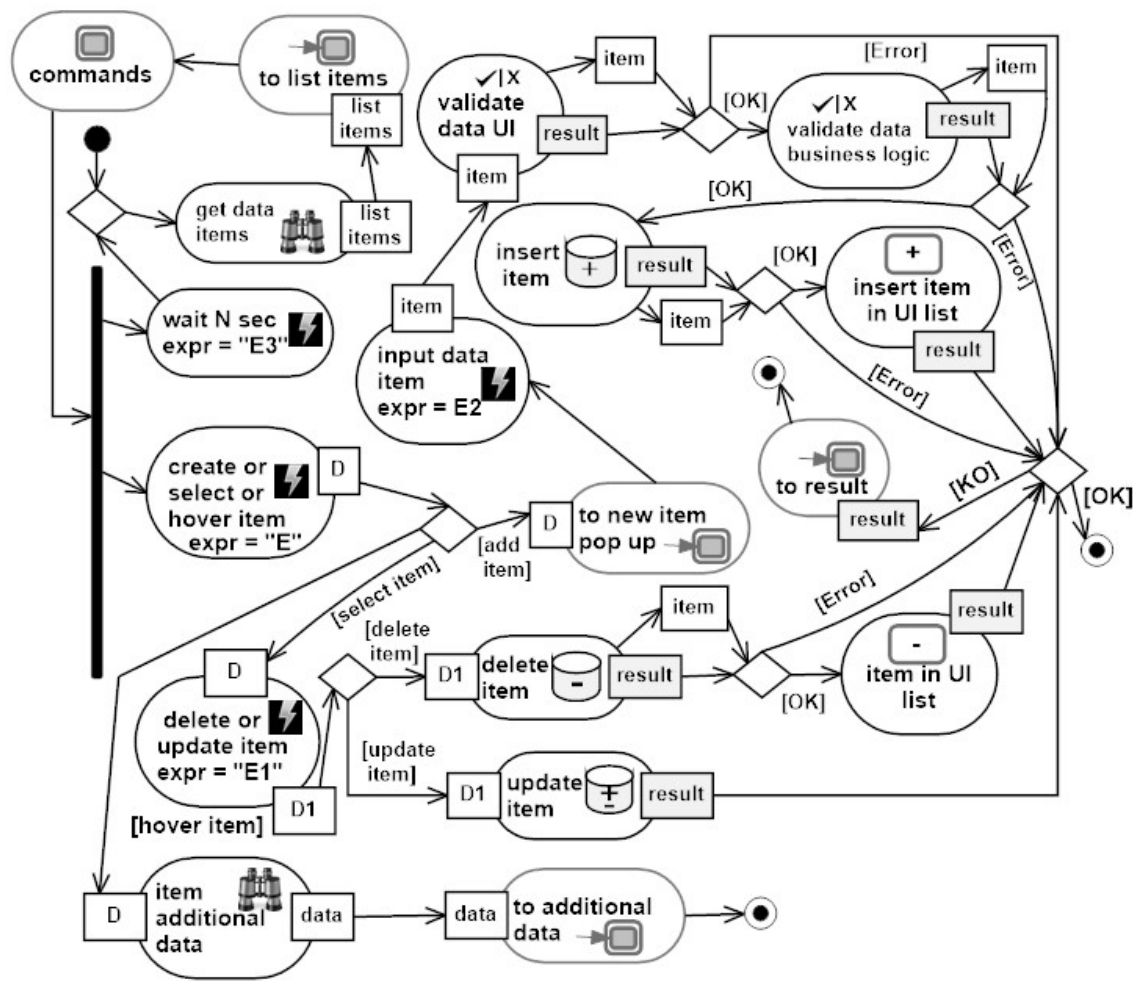

Fig. 6. UIUPDM for enriched CRUD of items of a list use case 
Fig.6 shows an example of an UIUPDM model for case a), for enriched CRUD of items of a list case study; $E$ and $E_{1}$ are defined in Fig. 4, $E_{2}$ is the identifier of event Press on submit of new item pop up and $E_{3}$ is the identifier of event timeout $N$ sec on timer. In addition, we use «synch» to notification action to obtain a result notification from creation, delete and update item operations results; «synch» to list items action is used to present the list of items CS and «present» commands is used to present the commands UiInputStructure.

For the automatic generation of UIUPDM we consider transformations defined in ATL language that map requirements for UCs and trace relations for these ADs onto a UIUPDM by applying some rules. For explaining transformation rules we used the following nomenclature: IP for inputPin, OP for outputPin, a.name for the name of element $a, n \diamond n$ ' to say that node $n$ is related to node $n$ ' in its $\mathrm{AD}$ (related means either an activityEdge or a Pin association); $a$ : outputAction means $a$ is either of stereotype «output content», or «output message», or «output media», or «output request»; $a$ : ForJobDesc means that $a$ is a RIAAD stereotype used for describing jobs; IPar means an input parameter node; OPar means an output parameter node. We consider the following operations from activity edges to activity nodes: $-(e)$ returns the origin of $e$ and $+(e)$ returns the target of $e$. Finally, [p: IP $\diamond$ ] $a$ means that it optionally exists an input pin $p$ of $a$ (the similar meaning for $a$ [१: OP]).

In Table 2, rule 4/5 transforms an «output request»/«output message» element without input pins and with a trace to an UIE $u$ onto a «present» u.name element; rule 6 maps an outputAction element with input pins and a trace into an UIE $u$ into a «synch» to u.name element; rules from 7 to 12 are used for copying into the result the nodes and edges of an AD describing an autonomous action of the input AD model; rule 7 copies the action nodes (and its pins if they exist) in the AD describing the job; rule 10 only copies the activity edges that are present in the $\mathrm{AD}$ describing the job action, and are not connected to parameter nodes of this AD. Rule 11 transforms the connection of an object node $i$ to a job described with an $\operatorname{AD} A$, onto the connection of object node $i$ to an activity node $n$, where $n$ is the node in $A$ that is connected with an input parameter with name $i$.

In Fig 6.with the help of the rules from 7 to 12, all actions and pins of the activity validate data and add item are copied, and if it is necessary such actions are connected to predecessor/successor actions of the job validate data and add item action in the activity containing this «job». The «synch» list items action is obtained by applying rule 6 to «output content» list items action.

Transformation AD\&Traces2UIUPDM_b) for case b) needs only the addition to rules from 1) to 7) of relationships from «present»/«synch» actions to UIEs. Transformation AD\&Traces2UIUPDM_c) for case c) uses an auxiliary function that returns true iff an UIE description is small that is used to decide inside rules from 1) to 7) if relationships from «present»/«synch» actions to UIEs are considered; and there is also an auxiliary function that returns true iff a job description is of small size that is used to decide if rules from 8) to 12) are applied for a job or not. 
Table 2. Transformation rules of AD\&Traces2UIUPDM case a)

\begin{tabular}{|c|c|c|}
\hline rule & Maps & Onto \\
\hline 1 & «input» a trace «event»e & «event» $\mathrm{e}$ \\
\hline 2 & «input and suggest» a trace «event»e & «event»e \\
\hline 3 & «search» a trace a': action & a': action \\
\hline \multirow[t]{2}{*}{4} & «output request» $a$ trace $u$ :UIE & \multirow[t]{2}{*}{ «present» u.name } \\
\hline & Condition: $a$ has not input pins & \\
\hline \multirow{2}{*}{5} & «output message» $a$ trace $u$ :UIE & \multirow[t]{2}{*}{ «present» u.name } \\
\hline & Condition: $a$ has not input pins & \\
\hline 6 & p: IP $\rightarrow$ a: outputAction trace $u:$ UIE & p: IP $\rightarrow$ «synch» to $u$.name \\
\hline \multirow{2}{*}{7} & [p: IP $\rightarrow$ ] a: ForJobDesc [ $\rightarrow p^{\prime}:$ OP] & \multirow[t]{2}{*}{ [p: IP $\rightarrow]$ a: ForJobDesc $\left[\rightarrow p^{\prime}: O P\right]$} \\
\hline & Condition: «job» a trace $\mathrm{A}: \mathrm{AD} \wedge \mathrm{a}$ in $\mathrm{A}$ & \\
\hline \multirow{2}{*}{8} & $o:$ objectNode & \multirow[t]{2}{*}{$o:$ objectNode } \\
\hline & $\begin{array}{l}\text { Condition: «job» a' trace } \mathrm{A}: \mathrm{AD} \wedge \\
o \text { in } \mathrm{A} \wedge o \text { is not a Pin }\end{array}$ & \\
\hline \multirow{2}{*}{9} & $n:$ controlNode & \multirow[t]{2}{*}{$n:$ controlNode } \\
\hline & Condition: «job» a' trace $\mathrm{A}: \mathrm{AD} \wedge n$ in $\mathrm{A}$ & \\
\hline \multirow{2}{*}{10} & $f$ : activityEdge & \multirow{2}{*}{$\begin{array}{l}f \text { : activityEdge } \\
-(f)=\mathrm{n}, \wedge+(f)=\mathrm{n}^{\prime}\end{array}$} \\
\hline & $\begin{array}{l}\text { Condition: «job» a' trace A: AD } \wedge \\
-(f)=\mathrm{n} \wedge+(f)=\mathrm{n}^{\prime} \wedge \mathrm{n}, \mathrm{n}^{\prime}: \text { activity-Node } \\
\wedge f, \mathrm{n}, \mathrm{n}^{\prime} \text { in } \mathrm{A} \wedge f \text { is not connected with a } \\
\text { parameter node }\end{array}$ & \\
\hline \multirow{2}{*}{11} & i: $\mathrm{ON} \rightarrow$ «job» a’ trace $\mathrm{A}: \mathrm{AD}$ & \multirow[t]{2}{*}{ i: $\mathrm{ON} \rightarrow \mathrm{n}:$ activityNode } \\
\hline & $\begin{array}{l}\text { Condition: i: IPar } \rightarrow \mathrm{n} \text { : activityNode } \wedge \mathrm{i}, \mathrm{n} \\
\text { in A }\end{array}$ & \\
\hline \multirow[t]{2}{*}{12} & «job» a’ trace $\mathrm{A}: \mathrm{AD} \wedge \mathrm{a} \mathrm{a}^{\prime} \rightarrow \mathrm{o:} \mathrm{ON}$ & \multirow[t]{2}{*}{$\mathrm{n}:$ activityNode $\rightarrow$ o: ON } \\
\hline & $\begin{array}{l}\text { Condition: } \mathrm{n}: \text { activityNode } \rightarrow \mathrm{o}: \mathrm{OPar} \wedge \mathrm{o}, \mathrm{n} \\
\text { in } \mathrm{A}\end{array}$ & \\
\hline
\end{tabular}

\section{$6 \quad$ Related Work}

Table 3 shows a comparison of our approach with other methods we found in the literature. Before explaining this table, we define some concepts: integrated models are models integrating user interaction with the UI and the response of the system to these interactions; integrated models can be: based on ECA Rules - ECA -, state machines -SM-, or integration of UI use with process modeling, permitting the definition of ECA rules - UIUP -; role means either analyst or UI designer. The column with title "two roles involved" means that to construct the integrated model at least one role has to understand diagram aspects that concern the other role (this may lead to one role interfering/involving with the other or to understandability reduction). 
Table 3. Method Comparison

\begin{tabular}{|l|l|l|c|l|}
\hline Method & $\begin{array}{l}\text { Integrated } \\
\text { model }\end{array}$ & $\begin{array}{l}\text { Notation } \\
\text { type }\end{array}$ & $\begin{array}{l}\text { Two roles } \\
\text { involved }\end{array}$ & $\begin{array}{l}\text { Way of obtaining integral } \\
\text { model }\end{array}$ \\
\hline WebML & $\begin{array}{l}\text { Hypertext } \\
\text { model }\end{array}$ & $\begin{array}{l}\text { partially a } \\
\text { UIUP }\end{array}$ & Yes & $\begin{array}{l}\text { Semi-automatic from } \\
\text { BPMN, or manually }\end{array}$ \\
\hline OOH4RIA & $\begin{array}{l}\text { Orchesta- } \\
\text { tion model }\end{array}$ & SM & $\begin{array}{l}\text { Yes, to define } \\
\text { interaction } \\
\text { dependences }\end{array}$ & $\begin{array}{l}\text { Semi-automatic from OOH } \\
\text { presentation } \\
\text { and navigation models }\end{array}$ \\
\hline RUX & $\begin{array}{l}\text { Interaction } \\
\text { presentation }\end{array}$ & ECA & Yes & Manually \\
\hline OOHDM & ADV & SM & Yes & Manually \\
\hline OOWS 2.0 & Event rules & ECA & Yes & Manually \\
\hline MARIA & $\begin{array}{l}\text { Dialog } \\
\text { model }\end{array}$ & ECA & Yes & $\begin{array}{l}\text { Some guides to obtain it } \\
\text { from CTTs }\end{array}$ \\
\hline our approach & UIUPDMs & UIUP & No & $\begin{array}{l}\text { Automatic generation } \\
\text { from RIA requirements and } \\
\text { traces }\end{array}$ \\
\hline
\end{tabular}

Only two of the methods are UIUPs: WebML ([2]) only partially, and our approach. WebML does not consider event patterns; the extension of WebML for Ajax technology ([1]) considers elements for modeling only typical Ajax events, and the treatment of such events do not consider all kinds of actions necessary for RIA applications (they do not consider operations modifying the structure/content of UIEs). Only our approach considers the construction of integrated models, without the necessity of a role having to read and understand details corresponding to the other role; OOH4RIA ([3]) alleviates partially this problem, because it semi-automatically generates an orchestration model skeleton that needs to be completed with the definition of interaction dependencies that need the involvement of analysts, who needs to comprehend UI details. Our development process generates automatically a complete integrated model, using design decisions from UI designers and analysts (captured by traces); WebML generates first versions of hypertext models from BPMN models that probably need to be refined or modified by designers. ECA approaches tend to be the less complex, followed by SMs, because SMs (in Table 3) approaches, in addition to ECA rules, consider UIEs with some details, and also control flow details. An UIUP must have at least the complexity of ECA rules, because it must describe ECA rules; we consider WebML more complex than our UIUPDMs, because it considers all kind of aspects: UIEs details, autonomous action description, and some relations of UIEs with the data model.

\section{Conclusion}

Our UIUPDM models contemplate several RIA needs presented in [16]: data management, definition of complex autonomous actions, validation of information, event handling, UIEs abstracting several widgets, the work with multimedia content, permits data synchronization, UIEs content/structure modification, UIE presentation, 
the possibility of the participation of several actors in a UIUPDM of a functionality, the communication with external Jobs, definition of tasks/business processes.

Our UIUPDMs abstract from details concerning: the size and positioning of UIEs, style of UIEs showing content, platform details, and device details; such details should be considered during concrete design or implementation. Our UIUPDM notations do not consider: a) the container hierarchies inside pages (only basic UIEs are considered in trace relationships); these can be defined using RIAAD for UI presentation; to know how basic visible UI components inside a container/page dynamically change, read the UIUPDMs; b) if the following operations are performed in the client side or the server side: CRUD operations on data, event processing, and validation of data; this can be considered during concrete design, or by adding more details to UIUPDMs operations (i.e. using a tagged value source, whose values can be 'client' or 'server').

The necessity of looking at other diagrams to understand UIUPDMs in more depth can be mitigated if we consider UIUPDMs as maps: by clicking on non-detailed modeling elements, they are expanded by using movable popups containing their descriptions (the UIUPDM does not disappear after these expansions). For UIUPDM_a) «synch»/«present» actions can be expanded, for UIUPDM_b) «job» actions can be expanded, and for UIUPDM_c) not detailed «synch»/ «present»/《job» actions can be expanded. Building a tool supporting this idea is a task for the future.

To find RIAAD modeling elements, we have considered some case studies: the ones of URLs in Sec. 3, case studies of the next sentence, and the approaches for RIA in the literature. We have applied our development process to the following case studies: enriched collaborative CRUD of items of a list, image editing with auto-save, live feed update, hover detail, on demand scrolling, state information update, book return, and the e-Learning platform in www.parleys.com.

Concerning the problems that would arise if UIUPDMs are built without using a carefully designed development process, we have taken the following preventive measures against them: a) inconsistency introduction: analysts and UI designers respect: business process description by using traces to refine actions, and detailed data description during trace relationship definition; in addition, data and control flows of requirements are copied into UIUPDMs; b) the construction of UIUPDMs by analysts and/or UI designers: it is avoided, because UIUPDMs are generated automatically from trace relationships; c) complex UIUPDMs: we decided to abstract from several details (see the 2nd paragraph in this Sec.), and to have different kinds of UIUPDMs concentrating on some aspects and avoiding others.

The development of a tool supporting de development process of this paper is a task for the future.

The necessary effort during abstract design is at least the effort that takes building trace relationships; there is one trace relation per action in a UC AD; UIEs in trace relationships are UIEs necessary for performing input or output, and do not involve entire pages or complex container hierarchies; we think that the biggest effort is required by describing complex autonomous actions.

The process for obtaining UIUPDs does not depend on: a) the abstraction level of UI design elements and event patterns; b) the particular set of UI design elements. 


\section{References}

1. Brambilla, M., Fraternali, P., Molteni, E.: A Tool for Model-driven Design of Rich Internet Applications based on AJAX. In: Murugesan, S. (ed.) Handbook of Research on Web 2.0, 3.0, and X.0: Technologies, Business, and Social Applications, ch. 31, pp. 96-118. IGI Global (2010) ISBN: 9781605663845

2. Fraternali, P., Comai, S., Bozzon, A., Toffetti, G.: Engineering rich internet applications with a model-driven approach. ACM Trans. Web 4(2), Article 7, 47 pages (2010)

3. Melia, S., Gomez, J., Perez, S., Diaz, O.: A Model-Driven Development for GWT-Based Rich Internet Applications with OOH4RIA. In: Proceedings of the 8th Intl. Conf. on Web Engineering, pp. 13-23. IEEE (2008)

4. Urbieta, M., Rossi, G., Ginzburg, J., Schwabe, D.: Designing the Interface of Rich Internet Applications. In: Proccedings of Latin American Web Congress 2007, pp. 144-153. IEEE (2007)

5. Linaje Trigueros, M.: Rux-method: modelado de interfaces de usuario Web multidispositivo, multimedia, interactivas y accesibles. Sánchez Figueroa, F. (dir.) (2009)

6. Valverde Giromé, F.: OOWS 2.0: Un Método De Ingeniería Web Dirigido Por Modelos Para La Producción De Aplicaciones WEB 2.0. Pastor López, O. (dir.) (2010)

7. Paternò, F., Santoro, C., Spano, L.D.: MARIA: A universal, declarative, multiple abstraction-level language for service-oriented applications in ubiquitous environments. ACM Trans. Comput.-Hum. Interact. 16(4), 1-30 (2009)

8. Bry, F., Eckert, M., Pătrânjan, P.-L., Romanenko, I.: Realizing business processes with ECA rules: Benefits, challenges, limits. In: Alferes, J.J., Bailey, J., May, W., Schwertel, U. (eds.) PPSWR 2006. LNCS, vol. 4187, pp. 48-62. Springer, Heidelberg (2006)

9. Eckert, M., Bry, F., Brodt, S., Poppe, O., Hausmann, S.: A CEP Babelfish: Languages for Complex Event Processing and Querying Surveyed. In: Helmer, S., Poulovassilis, A., Xhafa, F. (eds.) Reasoning in Event-Based Distributed Systems. SCI, vol. 347, pp. 47-70. Springer, Heidelberg (2011)

10. Casalánguida, H., Durán, J.E.: Requirements Engineering of Web Application Product Lines. In: Proceedings of the 7th Intl. Conf. on Web Information Systems and Technologies, pp. 418-425. SciTePress (2011)

11. Koch, N., Knapp, A., Zhang, G., Baumeister, H.: UML-Based Web Engineering. An Approach Based on Standards. In: Web Engineering: Modelling and Implementing Web Applications. Human Computer Interaction Series, pp. 157-191. Springer (2008)

12. Filho, O., Ribeiro, J.: UWE-R: An Extension to a Web Engineering Methodology for Rich Internet Applications. WSEAS Trans. Info. Sci. and App. 6(4), 601-610 (2009)

13. Mbaki, E., Vanderdonckt, J., Guerrero, J., Winckler, M.: Multi-level Dialog Modeling in Highly Interactive Web Interfaces. In: Proceedings of the 8th Intl. Conf. on Web Engineering, pp. 44-49. IEEE Computer Society, Los Alamitos (2008)

14. Bry, F., Pătrânjan, P.: Reactivity on the Web: Paradigms and Applications of the Language XChange. J. Web Eng. 5(1), 3-24 (2006)

15. Manolescu, I., Brambilla, M., Ceri, S., Comai, S., Fraternali, P.: Model-driven design and deployment of service-enabled web applications. ACM Trans. Internet Techn. 5(3), 439-479 (2005)

16. Toffetti, G., Comai, S., Preciado, J.C., Linaje Trigueros, M.: State-of-the Art and trends in the Systematic Development of Rich Internet Applications. J. Web Eng. 10(1), 70-86 (2011) 